\title{
Methodology to identify dwarfing gene d60 that complements gamete lethal gene gal by Next-generation DNA sequencing analysis
}

\section{Author:}

\section{Motonori Tomita}

Research Institute of Green

Science and Technology,

Shizuoka University

836 Ohya, Suruga-ku, Shizuoka

422-8529, Japan

E-mail:

tomita.motonori@shizuoka.ac.jp
Keywords: genomics,

Next-Generation Sequencer, isogenic genome

\begin{abstract}
The author previously discovered dwarfing gene, d60, and it complements the gametic lethal gene, gal, to cause gametic lethality in rice. Namely, in the F1 hybrid (genotype D60d60Galgal) of Koshihikari (D60D60galgal) $\times$ Hokuriku 100 (d60d60GalGal), male and female gametes having both gal and d60 become gametic lethal and the pollen and seed fertility decrease to $75 \%$. Therefore, F2 progeny shows a unique mode of inheritance that is segregated into a ratio of 6 fertile long-culm (4D60D60: 2D60d60GalGal) : 2 partially fertile long-culm (D60d60Galgal $=$ F1 type $): 1$ dwarf (d60d60GalGal).Prior to Next-Generation
\end{abstract} Sequencing analysis targeting d60, it is required to develop isogenic genome of reference except for target d60 gene. When the F1 (D60d60Galgal) progenies of 'Koshihikari' $\times$ 'Hokuriku 100' were backcrossed to 'Koshihikari' (D60D60galgal), BC1 F1 individuals segregated in a ratio of 1 tall and 25\% sterile (D60d60galGal) : 2 tall (1 D60D60Galgal : 1 D60D60galgal). Here, D60d60Galgal-heterozygous plants can be recognized by pollen sterility prior to anthesis. Then Tall and $25 \%$ sterile BC1F1 plants (D60d60galGal) were selected by $25 \%$ pollen sterility and backcrossed with 'Koshihikari' as female parent to produce $\mathrm{BC} 2 \mathrm{~F} 1$ seeds. Hereafter, D60d60Galgal-heterozygous plants were selected in the first generation of each backcross $(\mathrm{BCnF} 1)$ and immediately backcrossed repeatedly with Koshihikari to obtain isogenic genome useful for next-generation sequencing. That is to say, the target d60 DNA region narrowed down with each back cross generation, enabling identification of the target DNA mutation point by whole genomic sequencing. 


\section{Introduction}

The breeding program that has made the greatest contribution in the history of mankind is the 'Green Revolution,' in which the production of rice and wheat grain was dramatically increased in the 1960s with the development of dwarf varieties (Khush 1999). Dwarfing prevents plants from lodging at their full-ripe stage, makes them lodging-resistant to wind and rain, has enhanced their adaptability for heavy manuring and has dramatically improved the productivity up to double in rice and quadruple yields in wheat. In recent years, dwarf genes, which have been used as breeding materials to improve lodging-resistance, have been isolated. $s d l$, which is a dwarf gene that contributed to the 'Green Revolution' of rice, is a defective C20-oxidase gene in a late step in the gibberellin (GA) synthesis pathway (defective GA20-oxidase gene) (Sasaki et al. 2002). Surprisingly, rice semidwarf varieties developed independently by using different native varieties or artificially induced mutant lines as mother plants happen to be controlled by a same single dwarfing gene, $s d l$ (Sasaki et al. 2002). In addition, both $d 35$ from Tanginbouzu, which became the best rice cultivar in Japan between 1955 and 1964, and d18 from Kotake-tamanishiki were also kaurenoic acid oxidase- or 3-beta hydroxylase-defective in the same GA synthesis pathway (Itoh et al. 2001). On the other hand, Rht, which contributed to the 'Green Revolution' of wheat, is missing a gene involved in GA's signaling (Peng et al. 2000; Hedden 2003), and the Daikoku-type dwarf gene $d l$ in rice is defective in the alpha subunit of the heterotrimeric $G$ protein, affecting GA signal transduction (Ueguchi-Tanaka et al. 2000). Above all, available dwarf genes are limited to those associated with gibberellin (GA) for both rice and wheat.

Although dwarf varieties of rice have contributed to the dramatic improvement and stabilization of yields all over the world, $s d l$ is the world's only short-culm gene usable in actual rice breeding so far. Namely, options are limited. In consideration of the purpose to maintain/expand genetic diversity of plant 
cultivars, we should not rely on GA synthesis-defective gene $s d l$ and should discover more new dwarfing genes and promote their use in lodging-resistant breeding. Therefore, it is necessary to acquire a wide range of novel dwarfing genes in order to cope with climate changes.

\section{A unique genetic mode of novel} semidwarfing gene $d 60$

In order to find a novel dwarf gene that can take the place of the green revolution gene sd1, the author conducted gene analyses focusing on Hokuriku 100, which is a radiation-induced mutant line with culms approx. $15 \mathrm{~cm}$ shorter than those of variety Koshihikari (Samoto \& Kanai 1975), however, despite its promising phenotype, had been left aside because of unclear genetic mode. As a result, the author discovered a novel dwarf gene, d60, which brings about a good plant type with erect leaves by shortening culms by about 20\% (Tomita 1996). Furthermore, the author discovered that this dwarfing gene, $d 60$, and that it complements the gametic lethal gene, gal, to cause gametic lethality (Tomita et al 2003). Therefore, in the $F_{1}$ hybrid (genotype D60d60Galgal) of Koshihikari $($ D60D60galgal $) \times$ Hokuriku 100 (d60d60GalGal), male and female gametes having both gal and $d 60$ become gametic lethal and the pollen and seed fertility decrease to $75 \%$ (Fig.1). Namely, $\mathrm{F}_{2}$ progeny shows a unique mode of inheritance that is segregated into a ratio of 6 fertile long-culm (4D60D60: 2D60d60GalGal) : 2 partially fertile long-culm $\left(\right.$ D60d60Galgal $=\mathrm{F}_{1}$ type $)$ : 1 dwarf (d60d60GalGal). In other words, as the gametes with gal and $d 60$ result in death, $d 60$ is not transmitted to progeny without $\mathrm{Gal}$. Furthermore, isogenic lines that were developed by introducing both $d 60$ and $s d l$ genes into Koshihikari by backcrossing, namely the $d 60 s d l$ line, became additively extreme-dwarf (Tomita 2012), indicating demonstrating that $d 60$ is functionally independent from $s d l$ and not involved in the GA1 synthesis pathway.

\section{Genome analysis in Koshihikari}

\section{genetic background}

The threat of strong typhoons is 
increasing, probably as a result of global warming. This is a serious problem in rice production, because strong winds cause stem lodging and consequent yield losses and deterioration in crop quality. Koshihikari is the major rice cultivar and has approximately $40 \%$ of crop acreage in Japan. However, Koshihikari has shown a reduction in yield and loss of quality due to lodging and immature chalky grains, which have been caused by recent climate change due to global warming. Therefore, there is an urgent need to develop and disseminate a semidwarf Koshihikari variety that is resistant to lodging. Hence there is a pressing need to develop new short-culm rice cultivars resistant to strong winds. The genome wide association analysis was conducted in the rice genome by using target gene-substituted isogenic Koshihikari.

Today's advantage of genomics is developing Next-generation sequencer that is able to decode giga level of DNA sequences. Development of Next-Generation DNA sequencer is advanced under Obama care that aims to realize a societal implementation of medical genomics (Bahassi \& Stambrook
2014; Levy \& Myers 2016). NGS can read 15 million DNA fragments at one run (that are equivalent to 30 times of the rice genome which can be sequenced) and yielding high throughput, high-quality data. Prior to Next-Generation Sequencing, it is required to develop isogenic genome of reference except for target $d 60$ gene. Then the author crossed Koshihikari with cultivars with $d 60$ and then the offspring carrying the target gene $d 60$ was selected in the offspring and repeatedly backcrossed with Koshihikari. Except for $d 60$ gene region, the genome returned to Koshihikari as reference genome, every time backcross was repeated. When the $F_{1}$ (D60d60Galgal) progenies of 'Koshihikari' × 'Hokuriku 100' were backcrossed to 'Koshihikari' (D60D60galgal), $\quad \mathrm{BC}_{1} \quad \mathrm{~F}_{1}$ individuals segregated in a ratio of 1 tall and 25\% sterile (D60d60galGal) : 2 tall (1 D60D60Galgal : 1 D60D60galgal) (Fig. 1). Here, D60d60Galgal-heterozygous plants, can be recognized by pollen sterility prior to anthesis. Then Tall and $25 \%$ sterile $\mathrm{BC}_{1} \mathrm{~F}_{1}$ plants (D60d60galGal) were selected by $25 \%$ pollen sterility and backcrossed with 
'Koshihikari' as female parent to produce $\mathrm{BC}_{2} \mathrm{~F}_{1} \quad$ seeds. Namely, D60d60Galgal-heterozygous plants were selected in the first generation of each backcross $\left(\mathrm{BC}_{n} \mathrm{~F}_{1}\right)$ and immediately backcrossed repeatedly with Koshihikari to obtain isogenic genome useful for next-generation sequencing (Fig. 1). That is to say, the target $d 60$ DNA region narrowed down with each back cross generation, enabling identification of the target DNA mutation point by whole genomic sequencing (Fig. 2). This process is called genome-wide association analysis. Using the high-throughput, long-read next-generation sequencer, a library of 15-20 million reads (insert length $500 \mathrm{bp}$ ) was set up from $2 \mathrm{Gp}$, covering the rice genome size of over $400 \mathrm{Mb}$ $\mathrm{x}$ 30. Clusters then were formed on flow cells and pair-end sequencing of 250-bp-long read was performed. The read sequences were mapped on the reference genome of Koshihikari. Thus, introgression of target genes and that the rest of the sequences are the same as the original becomes obvious at a glance by sequencing the entire genome via next-generation sequencer. Our process of genome wide association analysis would be expected to lead to the rapid completion of a cultivar that has the same genome of Koshihikari except for the integrated useful target gene, at the same time. 


\section{References}

Bahassi, E., Stambrook, PJ. (2014). Next-generation sequencing technologies: breaking the sound barrier of human genetics. Mutagenesis 29, 303-310.

Hedden, P. (2003). The genes of the Green Revolution. Trends in Genetics 19, 5-9.

Illumina (2016). Document \# 15027987 v01. Nextera ${ }^{\circledR}$ DNA Library Prep Reference Guide.

Itoh, H., Ueguchi-Tanaka, M., Sentoku, N., Kitano, H., Matsuoka, M., Kobayashi, M. (2001). Cloning and functional analysis of two gibberellin 3 beta-hydroxylase genes that are differently expressed during the growth of rice. Proc. Natl. Acad. Sci. USA 98, 8909-8914

Khush, GS. (1999). Green revolution: preparing for the 59321st century. Genome 42, 646-655.

Levy, SE., Myers, RM. (2016). Advancements in Next-Generation Sequencing. Annual Review of Genomics and Human Genetics 17, 95-115

Peng, JR, ; Richards, DE., ; Hartley, NM.,
Murphy, GP., Devos, KM., Flintham, JE., Beales, J., Fish, LJ., Worland, AJ., Pelica, F., Sudhakar, D., Christou, P., Snape, JW., Gale, MD., Harberd, NP. (1999). 'Green revolution' genes encode mutant gibberellin response modulators. Nature 400, 256-261.

Samoto, S. \& Kanai, D. (1975). Studies on the mutation breeding in rice. I. Short stiff mutations induced by gamma-ray irradiation to the rice variety Koshihikari. Japanese Journal of Breeding 25, 1-7.

Sasaki, A., Ashikari, M., Ueguchi-Tanaka, M., Itoh, H., Nishimura, A., Swapan, D., Ishiyama, K., Saito, T., Kobayashi, M., Khush, G. S., Kitano, H. \& Matsuoka, M. (2002). Green revolution: a mutant gibberellin650synthesis gene in rice. Nature 416, 701-702.

Tomita, M. (1996). The gametic lethal gene gal: activated only in the presence of the semidwarfing gene $d 60$ in rice. In Rice Genetics III (ed. G. S. Khush), pp. 396-403. Manila: International Rice Research Institute.

Tomita, M. (2012). Combining two 
semidwarfing genes $\mathrm{d} 60$ and $\mathrm{sd} 1$ for reduced height in 'Minihikari', a new rice germplasm in the 'Koshihikari'genetic background. Genetics Research, Cambridge, 94, 23 -244.

Tomita, M., Yamagata, H. and Tanisaka, T.
(2003). Developmental cytology on the gametic abortion caused by the induced complementary genes, gal and d60, in Japonica rice. In Advances in Rice Genetics (eds. G.S. Khush, D.S. Brar and B. Hardy), pp.178-181. Manila: International Rice Research Institute. 


\section{Figures:}

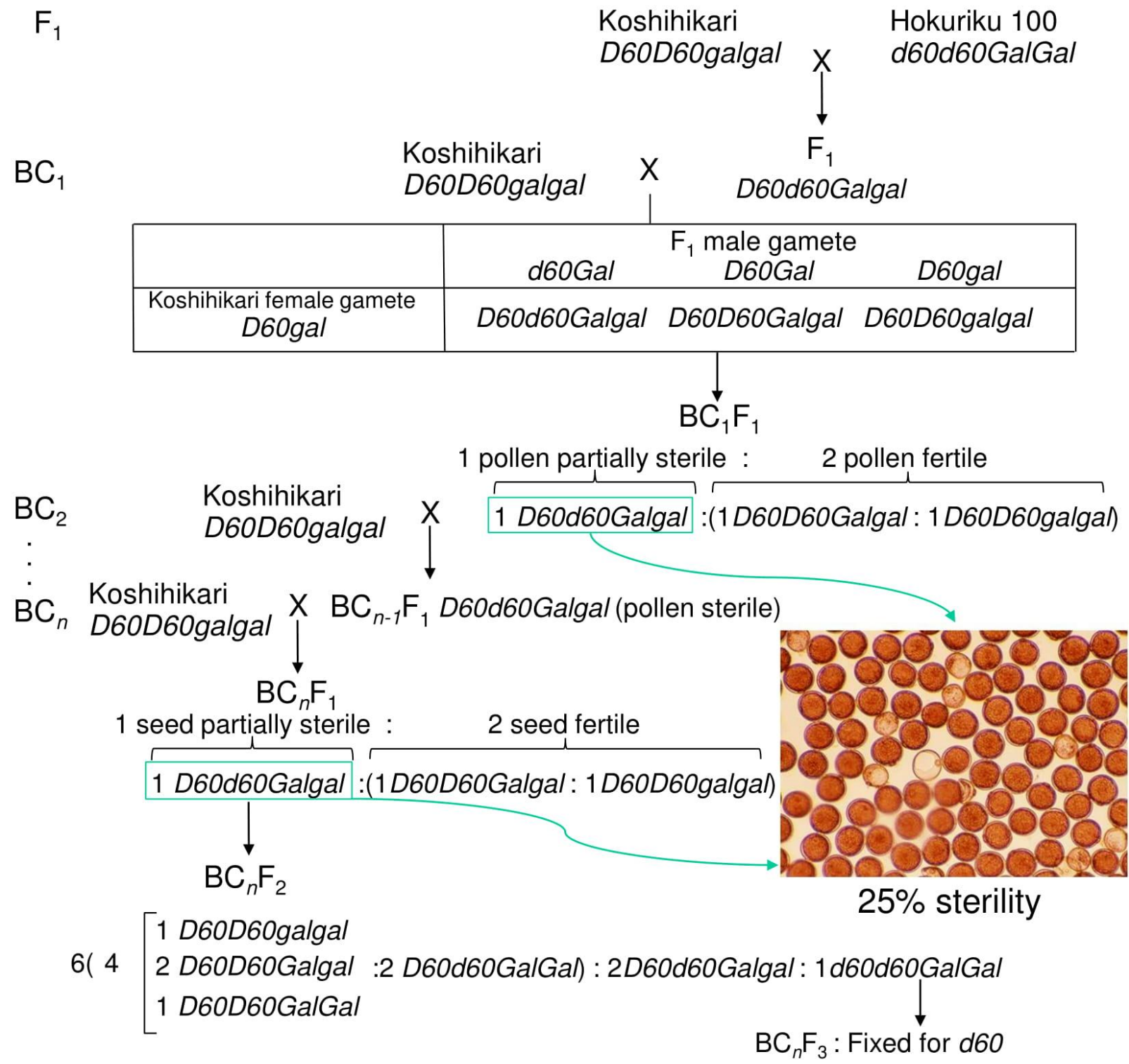

Fig. 1. Back-cross introgression of semidwarfing gene d60 by using $D 60 d 60 G$ algal-heterozygous $\mathrm{BC}_{n} \mathrm{~F}_{1}$ plants as male parent to obtain isogenic genome, which is devised from Tomita (2012), for whole genomic Nextgeneration sequencing 
Medical Research Archives, Vol. 7, Issue 6, June 2019

Methodology to identify dwarfing gene d60 that complements gamete lethal gene gal by Next-generation DNA sequencing analysis

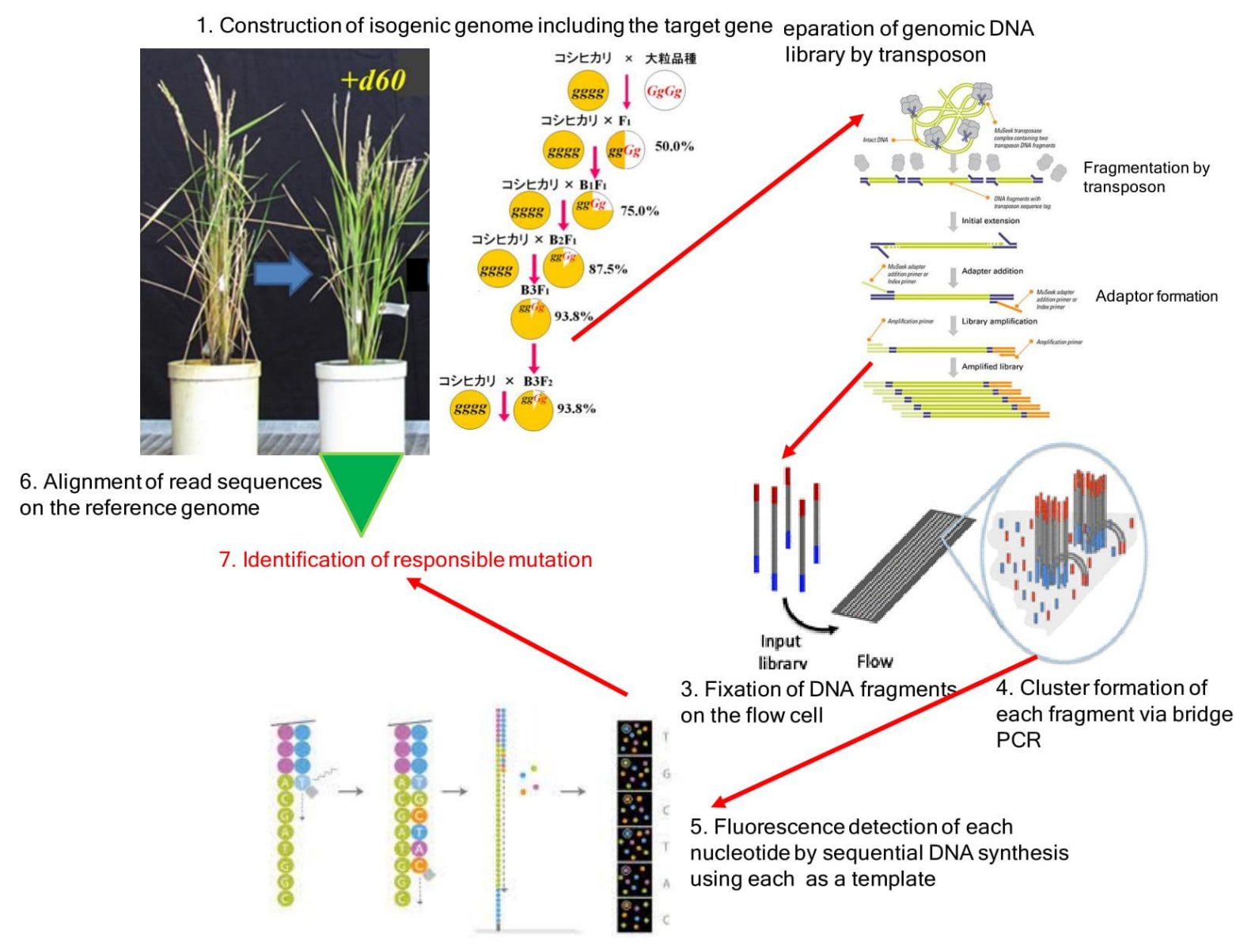

Fig. 2. Next-generation sequencing analysis of isogenic genome to identify responsible gene by using Illumina system (2016) 